\title{
A ACETAMIDA DE PIRROLIDONA COMO MEDICAÇÃO AUXILIAR NO TRATAMENTO DA PARALISIA CEREBRAL
}

\author{
Francisca Salete Pinheiro Chagas Ribeiro* \\ PENILdon Sillva** \\ Maria Lúcia Villas Boas DORIa *** \\ AlZA DE OliveIra LIMA $* \pi * *$ \\ Marisa Prates Campos $* * * * *$ \\ ANTONIETA NovaES Ferreira ****** \\ Maria Júlia Passos Rocha Santos ****** \\ REgina LÚCIA Ávila ****** \\ IONE DA Silva MaRTINS $* * * * * *$
}

$\mathrm{Na}$ história natural da paralisia cerebral (PC), chama a atenção a dificuldade do seu tratamento Para este fato contribuem não só numerosas variáveis, inclusive de ordem sócio-econômica, como também o caráter irreversível de certas lesões do sistema nervoso central que caracterizam a doença. Não é, portanto, de estranhar o interesse que vem despertando a abordagem bioquimica na terapêutica da PC, até agora predominantemente fisioterápica e pedagógica. Nessa modalidade de tratamento foi introduzido, no Brasil, por Low, Mello e Henriques (1974) ${ }^{16}$, o emprego da acetamida de pirrolidona ou piracetam. As propriedades farmacológicas do piracetam justificam seu ensaio terapêutico na $\mathrm{PC}$, pois se trata de molécula de ação preferencial nos neurônios telencefálicos, protegendo-os contra os efeitos da anóxia e de alterações metabólicas.

No presente trabalho, além da retificação dos resultados apresentados pelos autores supra mencionados, acrescentamos observações iniciais do efeito do piracetam sobre a espasticidade e testamos a tolerância da droga em nova apresentação, isto é, sob a forma de solução para uso oral, o que representa vantagem na administração do medicamento a crianças.

\section{MATERIAL E METODOS}

Foram observados 40 pacientes com diagnóstico de PC, nos quais visamos a observar a espasticidade, a neurolabilidade (agressividade, irritabilidade) e problemas de aprendizagem que interfiriam no rendimento da fisioterapia, da escolaridade e da terapia ocupacional.

Trabalho realizado no Instituto Bahiano de Reabilitação, apresentado no Vr Congresso Brasileiro de Paralisia Cerebral (Salvador, BA, 12 a 17 de outubro de 1975): * Fediatra, Diretora Técnica do Instituto Bahiano de Reabilitação; ** Titular de Farmacologia do Instituto de Ciencias da Saúde da Universidade Federal da Bahia; *** Psicopedagoga; ****Fisioterapeuta especialista em Bobath; ***** Fisioterapeuta; $* * * * *$ Professora especializada em excepcionais 
(O) fin ientes foram divilitios em dois grupos: grupo medicalo de 20 doentes aos quals, alenll de tratamenty rotiletro, fol administrada a droga, durante 10 semanas, c um yiup cuntrole, de parientes que só receberam tratamento rotineiro. Este tratamento rotinelro constou de tiés aspectos: clinico geral, fisloterápico e educacional enr classes para alunos excepcionais. No grupo mediculo, 10 paclentes foram submetldos a tratamento fisioterápico tijo Bobath e os outros 10 à fisioterapla de exercicios ciássicos.

A distribulcăo por sexo fol a seguinte: no grupo medicado, 12 eram do sexo mascultno e 8 do feminino; no grupo controle, 10 eram do sexo masculino e 10 do feminino. Quanto Idade a distribuição fol a seguinte: no grupo medicado: um paciente com 11 meses, 1 com 2 anos, dois com 3 anos, 5 cum' 5 anos, um com 6 anos, um com 8 anos, um com 9 anos, 4 com 10 anos, dois com 11 anos, um com 12 anos um com 13 anos; no grupo controle, 5 pacientes tinham 9 anos, 6 tinham 10 anos, 8 tinham 12 anos a um tinha 14 unos.

Os pacientes, selecionados previamente por fisiatras, neurologistas e psicologos, foram observados sob os seguintes aspectos: 1) exame psicológico, antes e depois da medicaçāo, verificando-se a atenção, o interesse, a concentração, a assimilação de instruçóes, a reacăo a estimulos, a Irritabilidade, a impulsividade, a hipersenstbilidade, o Q.I.; 2) acompanhemento clintco pela pediatra e pelas (isioterapeutas; 3) acompanhamento pedagóglco pelas professoras especializadas, nas classes para excepcilunals.

O piracetam fol administrado na dose de $80 \mathrm{mg} / \mathrm{kg}$ por dia, durante 10 semanas. A forma farmacêutica fol bem aceita por todos os pacientes. A droga, apresentada em solucão a $6 \%$ para uso oral *, não produziu eteitos colaterais, a não ser em um 'aso, no qual ocorreram vômitos somente nos primeiros dias da medlcacão. A toleIancia à droga mereceu cuidado especial, semclo verificada diariamente, no tocante aos principais sistemas e aparelhos.

\section{RESULTADOS}

Na avaliação clinica e pedagógica do grupo medicado 120 parientes) com piracetam, ao lado do tratamento clássico de PC (fisioterapla, terapia ocupacional, escolarizacão especializada), observou-se o resultado global seguinte: melhorados $65 \%$; inulterados 30\%; piorados $5 \%$.

Nesta avaliaçao, os mesmos parâmetros dos testes psicolóğicos foram seguidos obedecendo-se a uma escala de avallação assim discriminada: 1.) Inferior - desempenho muito abaixo do padrão das criansas observadas, tomaludo também a lactle como referéncia; 2) Médio inferior - desempenho não atinge a média das crianças observadas; 3) Médio - realizaçâo adequada is uma ldade cronológica e à méclla das crlanças observadas; 1) Médio superior - desempenho arima da média das criancas observadas; 5 l Superior - desempenho bem acima do padrāo dus (riancas observadas.

Nesta parte também se procurou observar a possivel influência da droga na espasticidade. Apesar de se saber que em qualquer resposta peisquisada nesse setor há necessidade de longo tempo de observaçäo, os resultados inlitais observados merecem registro. A melhora da espasticidade em 10 parientes no grupo medlcado, verifliadia năo só pela especialista em Bobath como pela fisioterapeuta responsável pejos vutros exerciclos, apjesentou o seguinte resultado: melhora acentuada em 20\%; melhora significativa $60 \%$; inalterados $20 \%$. No grupo controle a melhoria sí atinglu a $15 \%$.

Esses dados podem ser interpretados como resultantes da acão favorável sobre a atençāo, concentraşão e malor estabilidade emociunal das crialısas durante os exercicios. Os exercicios bem executados pelos paclentes irlam bloqueal a espasticidacle e melhorar o tonus muscular. E possivel que a droga possa favorecer a manutencão la aprendizagem dos estimulos usados na técniı́a pisioterápica.

- Nootropil Pediátrico - Rhodia, Divisão Falmacéutica. 
Grupo medicado com piracetam (20 pacientes)
Grupo controle nāo medicado (20 paclentes)

melhora inalterado piora melhora inalterado plora

\begin{tabular}{|c|c|c|c|c|c|c|}
\hline Atenção & $45 \%$ & $50 \%$ & $5 \%$ & $40 \%$ & $60 \%$ & $0 \%$ \\
\hline Interesse & $50 \%$ & $45 \%$ & $5 \%$ & $0 \%$ & $100 \%$ & $0 \%$ \\
\hline $\begin{array}{l}\text { Concentracão } \\
\text { Assimitacão de }\end{array}$ & $65 \%$ & $30 \%$ & $5 \%$ & $20 \%$ & $80 \%$ & $0 \%$ \\
\hline instrusōe!:! & $80 \%$ & $20 \%$ & $0 \%$ & $60 \%$ & $40 \%$ & $0 \%$ \\
\hline Q.I. & $60 \%$ & $20 \%$ & $20 \% *$ & $40 \%$ & $60 \%$ & $0 \%$ \\
\hline Respusta a estímulos & $70 \%$ & $30 \%$ & $0 \%$ & $10 \%$ & $90 \%$ & $0 \%$ \\
\hline Irritabilldade & $65 \%$ & $10 \%$ & $25 \%$ & $10 \%$ & $80 \%$ & $10 \%$ \\
\hline Impuisividade & $60 \%$ & $35 \%$ & $5 \%$ & $0 \%$ & $80 \%$ & $20 \%$ \\
\hline Hipersensibllidade & $30 \%$ & $60 \%$ & $10 \%$ & $0 \%$ & $80 \%$ & $20 \%$ \\
\hline
\end{tabular}

Quadio 1 - Resultados: * intestável

\section{COMENTARIOS}

Agindo na célula cerebral em sofrimento, seja este de que natureza for, - piracetam não pode ser considerado como especifico de qualquer condição neurológica. Trata-se, portanto, de medicação de fundo, de base, na patologia cerebral. No homem esta patologia varia desde as anomalias hereditárias até às causas traumáticas, vasculares, tóxicas, infecciosas, metabólicas, degenerativas e, até, afetivas. O piracetam vem sendo indicado especialmente nas perturbações das funções cerebrains no seu nível mais elevado, isto é nas esferas intelectual e da consciência.

Quanto à farmacocinética da droga, alguns dados já são bem conhecidos. Após a administração oral, sua absorção é praticamente completa, sendo rápida a sua entrada na corrente sanguínea. A meia-vida da droga no sangue é de 4 horas c meia, aproximadamente. Sua eliminação é feita quase que exclusivamente por via renal Do sangue, o piracetam penetra livremente na maioria dos órgãos. Quanto à penetração no sistema nervoso central, observa-se que a travessia da barreira hemato-encefálica se faz mais lentamente. A meia-vida do piracetam no líquido cefalorraqueano (LCR) è de 7 horas e meia, aproximadamente. A relação entre a quantidade do piracetam no LCR e no sangue cresce em função do tempo e ultrapassa a unidade Isto significa que, uma vez no interior do cérebro, a droga aí se mantém durante mais tempo do que no sangue. $O$ mecanismo de ação do piracetam no nivel da célula nervosa se traduz por consumo maior de glicose circulante e aumento da sintese da ATP a partir do ADP. Como é sabido, é a molécula do ATP que assegura, nas células vivas, a transferência de energia proveniente da glicose para as reações que consomem energia, como, por exemplo, os sistemas de biossintese de macro-moléculas (lipídios, proteinas, RNA) e os processos que mantêm a polarização da membrana celular. 
Essa estimulação das potencialidades energéticas das células nervosas, comprovada experimentalmente, é benéfica nos processos bioquímicos celulares deficitários, como se observa, por exemplo, nos organismos em hipóxia ou en. velhecidos

O piracetam, como medicação auxiliar no tratamento da PC, demonstrou ser útil, por melhorar as condições de rendimento pedagógico e de neurolabilidade das crianças, assim como a espasticidade em certo número de pacientes.

\section{RESUMO}

Vinte crianças, com diagnóstico de paralisia cerebral (PC) e sob tratamento clássico, fisioterápico e pedagógico, receberam piracetam (acetamida de pirrolidona) como medicação auxiliar. $O$ objetivo foi melhorar os problemas de espasticidade, apredizagem, neurolabilidade, visando a um rendimento melhor do tratamento global da PC. O grupo medicado foi comparado com um grupo controle de 20 crianças que só eram objeto do tratamento habitual. A comparação mostrou resultados favoráveis no grupo medicado. A droga foi administrada na dose de $80 \mathrm{mg} / \mathrm{kg} /$ dia durante 10 semanas. Os critérios de avaliação dos resultados foram psicológicos, clínico, fisioterápico e pedagógico $\mathbf{A}$ droga foi administrada em nova forma de apresentação: solução, para uso oral, a $6 \%$.

\section{SUMMARY}

\section{Pyrrolidone acetamide as an auxiliary drug in the treatment of cerebral palsy}

Twenty children, with the diagnosis of cerebral palsy (CP) and under classical, physiotherapeutical and pedagogical, treatment, received piracetam (pyrrolidine acetamide) as an auxiliary drug. The goal was to better spasticity, learning and nervous instability problems aiming at better results of over-all treatment of CP. The group that received the drug has been compared to a control group of 20 children treated by the customary treatment only. The comparison showed favourable results for the medicated group. The drug was administered in the dose of $80 \mathrm{mg} / \mathrm{kg} /$ day during 10 weeks. The criteria for evaluation have been psychological, clinical, physiotherapeutical and pedagogical. The drug has been given in a new form of presentation: $6 \%$ solution for oral use.

\section{REFERENCIAS}

1. AMPhOUX, G.; NONNAURE, C.; NORlOCK, B. \& SAGNES, J. - Etude preliminaire de l'action de I'UCB 6215 sur troubles de la conscience des traumatismes craniens et des accidents vasculaires cérébraux. An. Congrès de Psychiatrie et de Neurologie de Langue Françatse, Caen, 5-10 juillet, 1971.

2. BARTRAM, J. B. - Cerebral Palsy. In EMERSON, WALDO E.; VAUGHAM, V. C. \& MCKAY, R. J. - Textbook of Pediatrics. W. B. Saunders Co. Philadelphia, 1969 . 
3. BERTRAND, C. - Intérêt du piracetam comme correcteur de la dysmnesie consecutive à la sismothérapie. An Congrè de Psychiatrie et de Neurologle de Langue Française, Caen, 5-10 juillet, 1971.

4. BOBATH, $\mathrm{K}$. \& BOBATH, B. - The diagnosis of cerebral palsy in infancy. Arch. Dis. Childhood 31:408, 1956.

5. DURAD, H. \& SOLOMONOVICI, A. M. - Intérét de l'utilisation du piracetam (UCB 6215) dans les troubles de l'adaptation de l'enfant. Med. Infant. 78:478, 1971.

6. FERREY, G. \& BOUTIER, D. - Les syndromes subjectifs des traumatisés du crâne vus dans un service d'hôspital général en un an: utllisation de I'UCB 6215. Therapeutique $48: 143,1972$.

7. GIURGEA, C.; MOYERSOONS, F. \& EVRAERD, A. C. -- A gaba-related hypotesis on the mechanism of action of the antimotion sickness drugs. Arch. Int. Pharmacodyn. Ther. 166:238, 1967.

8. GIURGEA, C.; MOURAVIEFF-LeSUISSE, F. \& LEEMANS, R. - Correlations electro-pharmacologiques au cours de l'anoxie oxyprive chez le lapin en respiration libre ou artificielle. Rev. Neurol. (Paris) 122:484, 1970.

9. GIURGEA, C. E. \& MOYERSOONS, F. - Differential pharmacological reactivity of three types of cortical evoked potentials. Arch. Int. Pharmacodyn. Therap. $188: 401,1970$.

10. GIURGEA, C.; LEFEVRE, D.; LESCRENIER, C. \& DAVID-REMACLE, M. Pharmacological protection against hipoxia-induced amnesis in rats. Psychopharmacol. 20:160, 1971.

11. GIURGEA, C. \& MOURAVIEFF-LESUISSE, F. - Pharmacological studies on an elementary model of learning - the fixation of an experience at spinal level: part I: Pharmacological reactivity of the spinal cord fixation time. Arch. Int. Pharmacodyn. Ther. 191:279, 1971.

12. GIURGEA, C. \& MOURAVIEFF-LESUISSE, F. - Effect facilitateur du piracetam sur un apprentissage répetitif chez le rat. J. Pharmacol. 3:17, 1972.

13. GIURGEA, C. E. - Vers une pharmacologie de l'activité intégrative du cerveau: tentative du concept nootrope en psychopharmacologie. Acta Pharmacol. 25:115, 1972.

14. GOBERT, J: G. - Genèse d'un médicament: le piracetam; metabolisation et recherche biochimique. J. Pharmacie Belgique 27:281, 1972.

15. HOTERMANS, J. M.; VAN DAELE, G. \& SCHELlE KENS, K. - Etude pllote du pirrolidone acétamide ou piracetam en Neurologie et Neurochirurgie, portant sur 220 observations. An. Reunion Commune de Neurochirurgie, Bruxelles, juin, 1971.

16. LOW, R.; MELlo, P. A. \& HENRIQUES, F. G. - Ação do piracetam em criancas com paralisia cerebral Rev. Bras. Clin. Terap. 4:409, 1975.

17. MOURAVIEFF-LESUISSE, F. \& GIURGEA, C. E. - Pharmacological reactivity of an experimental model of memory: the spinal cord fixation. Arch. Int. Pharmacodyn. Ther, 176:471, 1968.

18. PEDE, J. P.; SCHIMPFESSEL, L. \& CROKAERT, R. - The action of piracetam on the oxidative phosphorylation. Arch. Int. Physiol. Bioch. 79:1036, 1971.

19. SARA, S. J. \& LEFEVRE, D. - Hypoxia-induced amnesia in one-trial learnIng and pharmacological protection by piracetam. Psycho-pharmacol. 25:32, 1972.

20. STRUBBE, J. H. \& CYPRYSIAK, E. - Dérivés de l'acide (2-oxo-pyrrolidine) acétique. Rev. Ind. Chim. Belge 32:112, 1967.

21. THIEBAULD, M. - Amélioration des performances intelectuelles. Contribution d'une thérapeutique corticale specifique. An. Congrès Français de Medecine, Beyrouth, 12-16 sept, 1971.

22. TOSCANO, M.; MENDLEWICZ, J. \& BOON, H. - Bilan de deux ans d'utilisation du piracetam (UCB 6215) en clinique psychiatrique. An. Congrès de Psychiatrie et de Neurologie de Langue Française, Caen, 5-10 juillet, 1971. 
23 TURON, R.; FI.ORENCE, J. \& PRAT, H. - Perspectives thérapeutiques de l'UCB 6215 - ptracetam - dans les états de détresse cérébrale aigùe, les séquelles dé traumatismes et leurs altérations électroencephalographlques. An. Congrès de Psychtatrie et de Neurologie de Langue Française, Caen, 5-10 Julllet, 1971.

:1. TURON, R. \& FLORENCE, J. - Résultats de l'expérimentation thérapeutique du plracetam (2-pyrrolldone acétamide) UCB 6215 dans les accidents vasculalres cérébraux (100 cas). An. Journées Int. de Circulation Cérébrale, Toulouse, 21-22 avrll, 1972.

25. WECKROTH, J. \& MIKKONEN, H. - Effet de l'UCB 6215 sur certains traits de performance intelectuelle, perceptuelle et psychomotrice et sur des tralts d'etats mentaux jugés subjectivement. An 30th Congres on Alcoholism and Drug Dependence, Amsterdan, 4-9 sept, 1972.

26. WOLTHUIS, O. L. - Experiments with UCB 6215 a drug which enhances acquisitions in rats: its effects compared with those of metamphetamine. Eur. J. Pharmacol, 16:283, 1971.

Instituto Bahiano de Reabilitacĩo - Av. Presilente Vargas - 40000 Salvador, BA-Brasil. 